\title{
Pflegeleistungen auswerten und vergleichen
}

\author{
Andreas Sund
}

\begin{abstract}
Mit INPULS ${ }^{\circledR}$ standen der Charité 2016 erstmals genaue Daten zum tatsächlichen Pflegeaufwand auf den Intensivstationen zur Verfügung. Im nächsten Schritt ging es darum, diese auszuwerten und miteinander zu vergleichen - um daraus letztendlich eine angemessene Personalbesetzung abzuleiten.
\end{abstract}

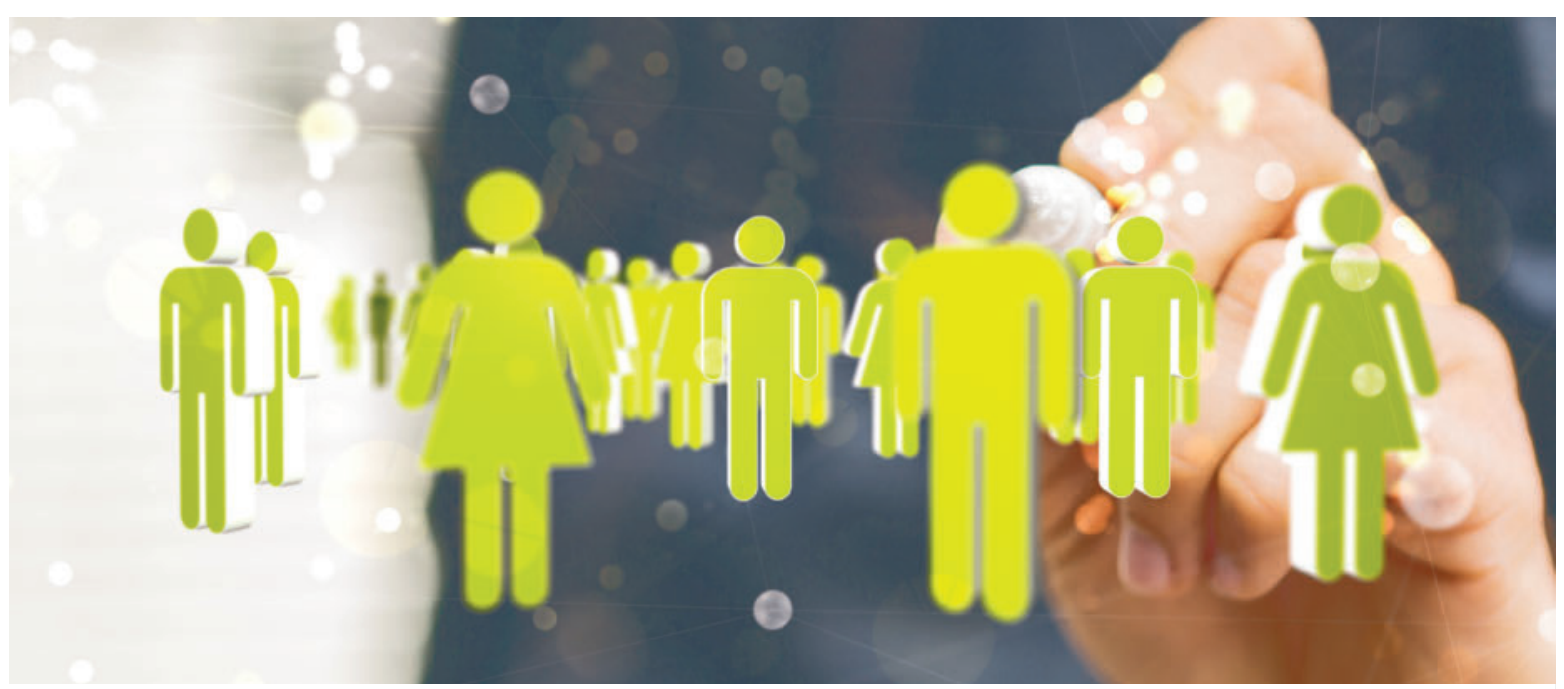

Nach der Auswertung der erfassten Daten lässt sich mithilfe des Leistungserfassungssystems eine Personalplanung realisieren, die sich an den tatsächlichen Bedarfen der Intensivstation orientiert. (Quelle: vegefox.com_AdobeStock)

Alle Intensivpflegeeinheiten der Charité - Universitätsmedizin Berlin nutzen nur noch ein Patientendatenmanagementsystem (PDMS), das fächerübergreifend identisch in seiner Oberflächenstruktur ist. Pflegeplanung und Dokumentation der durchgeführten Pflegemaßnahmen erfolgen einheitlich unter Nutzung der NANDA Pflegediagnosen, die verpflichtend anzuwenden sind. Die vier Punkte

- ein Dokumentationssystem

- vereinheitlichte PDMS-Struktur

- einheitliches Pflegeplanungstool

- standardisierte (modulare) Dokumentation im Popup-Design sowie Text- und Planungsbausteine (für alle im PDMS dokumentierenden Berufsgruppen)

waren entscheidende Voraussetzungen, um Pflegeleistungen in den Intensivstationen in gleicher Weise dokumentieren, ableiten, auswerten und miteinander vergleichen zu können.
Nach der Entscheidung zur Einführung des Leistungserfassungssystems INPULS ${ }^{\circledR}$ im Jahr 2015 wurden erste technische Schritte zur Integration in das bereits bestehende PDMS umgesetzt. Zur Abbildung der retrospektiv erhobenen Pflegekategorie (PK) ist zusätzlich ein Pflichtfeld zur Angabe des numerischen Werts und ein Zusatzfeld für die Begründung der Einstufung geschaffen worden. Dieses Feld wird von den Pflegenden jeweils nach Mitternacht (für den Vortag) oder zeitnah im Verlauf eines Behandlungstags, z. B. bei Verlegung, Entlassung oder Tod eines Patienten, ausgefüllt.

Der Einsatz eines elektronischen PDMS bietet die Möglichkeit der Ablage einer Vielzahl von pflegerisch und ärztlich erhobenen Daten, die in der täglichen Patientenversorgung anfallen. Somit sind ein direkter Zugriff und die Auslesung aller Daten zur zentralen Auswertung wesentlich leichter möglich als bei einer Papier-gestützten Dokumentation. 


\section{Erstmals Daten zum Pflegeaufwand auf den Intensivstationen der Charité}

Bis zur Einführung von INPULS gab es keine Möglichkeit, eine Aussage über reine Pflegeaufwände von Patienten der Intensivstationen der Charité zu treffen.

TISS (Therapeutic Intervention Scoring System), SAPS (Simplified Acute Physiology Score), SOFA (Sequential Organ Failure Assessment) etc. fanden und finden systematisch Anwendung und sind fester Bestandteil täglicher Erhebungen im Rahmen der Dokumentation, zur Beschreibung der Fallschwere, auf den Intensivstationen. Allerdings unterliegen sie alle einem Mangel, wenn sie zur Aufwandsmessung oder der Abbildung von Personalbedarfen herangezogen werden. Sie bilden pflegerische Leistungen nur indirekt oder gar nicht ab. Selbst bei separater Betrachtung von Zusatzaufwänden wie Transporten, Isolationsmaßnahmen, Mobilisations- und Transferhäufigkeiten etc. war eine Aussage zum Pflegeaufwand oder zu einer notwendigen pflegerischen Personalstärke pro Tag, pro Schicht oder pro durchschnittlichem Betrachtungszeitraum nur sehr eingeschränkt möglich. Aufwände zu Hygienemaßnahmen, Sterbebegleitung oder Angehörigenbetreuung fehlen gänzlich in der Betrachtung. Administrative Tätigkeiten, die zwangsläufig auf jeder Station, in jeder Versorgungs- und Pflegeeinrichtung stattfinden müssen, werden ebenfalls durch keines der oben genannten Systeme berücksichtigt. Sogenannte Stundenfälle, also Patienten, die nur für Stunden eine Intensivversorgung oder Überwachung benötigten, fielen in der Vergangenheit gänzlich durch die Raster der Scoring- und Aufwandsmesssysteme.

Nach der Einführung von INPULS war erstmals eine gesamthafte Darstellung der Intensivpatienten aller Intensivstationen der drei bettenführenden Standorte der Charité, unter Betrachtung von Pflegeaufwänden mittels einer zugeordneten Pflegekategorie (PK 1-6), möglich. Tatsächlicher Pflegeaufwand und erbrachte Pflegeleistung konnten in Zahlen dargestellt und vergleichend betrachtet werden.

Seit 2016 liegen für alle Intensivstationen die jeweiligen Pflegekategorien mit den dazugehörigen Pflegeminuten vor. Erstmals verfügt die Charité über valide Daten zum Pflegeaufwand und kann Intensiveinheiten im Detail miteinander vergleichen: Zahlen und Daten statt Emotionen und gefühlter Belastung.

\section{Schulungsmaßnahmen}

Für neue Kollegen und interessierte Mitarbeiter mit Schulungsbedarf bietet die zentrale Dokumentationsbeauftragte zu Beginn eines jeden Quartals im Jahr Schulungen zu den Themen Pflegedokumentation, administrativ-klinischer Arbeitsplatz und INPULS an. Zielgruppe sind alle Pflegenden der Intensivstationen der Charité und deren
Führungskräfte. Jede Schulung umfasst zwei Arbeitstage mit jeweils acht Stunden in einem Schulungsraum mit vernetzten PC-Arbeitsplätzen.

Regelmäßige Schulungsintervalle sind auch aufgrund von Überarbeitungen der Inhalte des INPULS-Katalogs nach den Anwendertreffen in Heidelberg notwendig. Hierzu werden die Schulungsinhalte angepasst und aktualisiert.

Im Rahmen von Arbeitstreffen des Qualitätszirkels Dokumentation werden die teilnehmenden Multiplikatoren über die Änderungen informiert. Zusätzlich wird der jeweils aktuelle INPULS-Katalog im Intranet der Charité abgelegt und ist für Anwender und Interessierte einsehbar.

\section{Aufbereitung und Interpretation der Daten}

Sämtliche Daten des PDMS der Intensivstationen lassen sich jederzeit mittels eines integrierten Tools durch die Leitungskräfte auslesen. Zusätzlich findet eine monatliche Aufbereitung der Daten durch das Controlling des Unternehmens statt. Sowohl der Pflegedirektion, den Centrumsleitungen und den Stationsleitungen werden diese Daten zum Zweck der Steuerung zur Verfügung gestellt.

Ein wesentlicher Bestandteil ist seit 2017 die gesamthafte Darstellung der erreichten Pflegeminuten nach INPULS für jede einzelne Intensivstation und der sich daraus ergebende durchschnittlich notwendige Personalbedarf der Pflege. Eine detailliertere Darstellung erfolgt zusätzlich, nach jedem abgeschlossenen Monat, durch den Intensivpflegekoordinator. Jeder Stations- und Centrumsleitung wird auf der Grundlage der Daten eine Empfehlung für die durchschnittliche Pflegepersonalbesetzung der einzelnen Schichten zur Verfügung gestellt. Schrittweise wird damit eine starre Schichtbesetzung durch eine orientierende Strukturbesetzung, die sich aufgrund durchschnittlicher Pflegeaufwände ergibt, ersetzt. Wichtig dabei ist, dass diese Strukturbesetzung dynamisch ist und monatlich nach oben oder unten angepasst werden kann.

\section{Überprüfung und Kontrollen}

Um valide Daten zur Einstufung der Patienten mittels einer Pflegekategorie und Aussagen über die Qualität der Dokumentation zu erhalten, musste eine Entscheidung zu Art und Umfang einer regelhaften Überprüfung getroffen werden. Jährlich finden zwei umfängliche Vor-Ort-Visiten im Vier-Augen-Prinzip mit direktem Feedback an die Stationsleitung oder ihre Stellvertretung statt. Des Weiteren zwei Fernvisiten durch Überprüfung der dokumentierten Daten im PDMS anhand eines festgelegten Algorithmus und mithilfe eines standardisierten Erfassungsbogens. Alle Kontrollen erfolgen unangekündigt. Teilnehmer der VierAugen-Prüfung sind jeweils die zentrale Dokumentations- 
beauftragte der Charité und eine Stationsleitung oder ein dezentraler Dokumentationsbeauftragter, die zwingend von einer anderen Intensivstation kommen müssen. Der eigene Bereich kann demnach niemals vom dort tätigen Personal selbst visitiert werden. Dieses Vorgehen entspricht üblichen Peer-Review-Verfahren.

Die Entscheidung, welche Intensivstation visitiert wird, erfolgt immer erst am Vorabend eines Visitentermins. Das Kontrollteam muss aufgrund des Umfangs der Daten zur Plausibilitätsprüfung am Tag der Visite komplett freigestellt sein.Die Fernprüfungen (Stichprobenprüfungen) finden jeweils mit dem zentralen Dokumentationsbeauftragten und dem Intensivpflegekoordinator statt. Umfang pro Jahr:

- 18 Intensivstationen (Erwachsene) mit COPRA 5 als PDMS

- 72 Überprüfungen insgesamt

- 2 Vor-Ort-Visiten + 2 Fernprüfungen pro Station

Die Ergebnisse der Plausibilitätsprüfungen werden elektronisch aufbereitet und den Leitungsteams, der jeweiligen pflegerischen Centrumsleitung und der Pflegedirektion übermittelt oder präsentiert. Die Zielerreichung für korrekt erhobene und plausible Pflegekategorien nach INPULS liegt bei 80 Prozent als Mindestanforderung.

\section{Nachsteuerung und Anpassung der Personalbesetzung}

Wesentliches Steuerungsinstrument zur Personalanpassung bei kurzfristigem Personalbedarf im Pflegebereich ist der Intensivpflegepool der Charité, der unter der Leitung des Intensivpflegekoordinators steht. Durch Kenntnis aller Intensivstationen, aller Dienstpläne und die Möglichkeit des Zugriffs auf alle elektronischen Patientenkurven ist eine Priorisierung der Unterstützung mit Pflegekräften aus dem Pool möglich. Insbesondere in Situationen, in denen mehrere Personalanforderungen von unterschiedlichen Intensivstationen vorliegen, die nicht alle bedient werden können. \ Abb. 1 zeigt eine vereinfachte Darstellung zum Prozess der kurzfristigen Vergabe.

\section{FAZIT}

Die Entscheidung für die Einführung von INPULS ermöglichte erstmals die Erfassung von reinen Pflegeleistungen. Dies war bislang nicht möglich. Heute können wir Aussagen zu Pflegeaufwänden treffen. Die Darstellung erfolgt transparent für alle Intensivstationen der Charité, die damit vergleichbar sind. Pflegepersonal ist nach tatsächlichen Bedarfen einsetzbar. Diese wiederum sind an Pflegeaufwand und dargestellte Pflegeleistung gekoppelt. Stufenweise etablieren wir eine durchschnittliche orientierende Strukturbesetzung, die den tatsächlichen

\section{Personalanforderungen Station A}

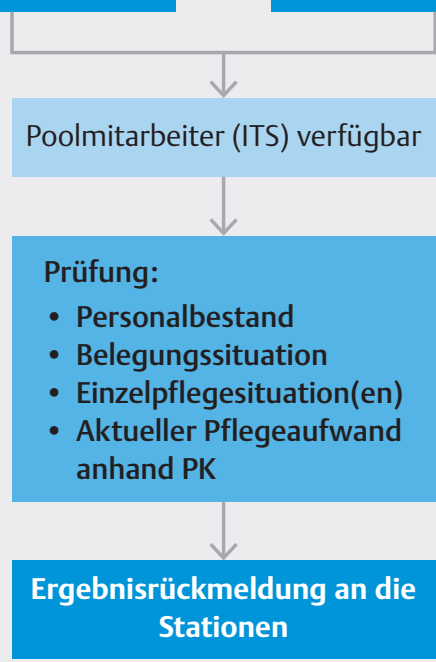

Abb. 1 Vergabeprozess.

und nachgewiesenen Pflegeaufwänden gerecht werden soll.

Ein weiterer positiver Nebeneffekt war die notwendige Angleichung der Dokumentation im PDMS. Sie ist heute, von wenigen Ausnahmen abgesehen, auf allen Intensivstationen identisch. Die Zuteilung von externem Personal, insbesondere von Mitarbeitern des Intensivpflegepools, orientiert sich nicht mehr an den pauschalen Anforderungen der einzelnen Stationen, sondern an festen Kriterien. Der Einsatz erfolgt prioritär und orientiert sich am Pflegeaufwand der aktuell auf einer Station befindlichen Patienten.

Autorinnen/Autoren

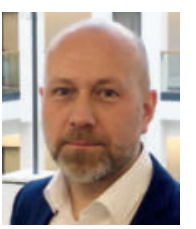

\section{Andreas Sund}

Intensivpflegekoordinator, Fachgesundheitsund Krankenpfleger für Anästhesie und Intensivpflege, Leiter des Intensivpflegepools und des Intensivpflege-Traineeprogramms, Charité - Universitätsmedizin Berlin. E-Mail: Andreas.Sund@charite.de

Bibliografie

DOI https://doi.org/10.1055/a-0821-3571

intensiv 2019; 27: 99-101

(c) Georg Thieme Verlag KG Stuttgart · New York

ISSN 0942-6035 